\section{Revista de Revistas (Journal Club de la Pontificia Universidad Católica de Chile)}

Intraoperative methadone for the prevention of postoperative pain. A randomized, doubleblinded clinical trial in cardiac surgical patients. Murphy GS, Szokol JW, Avram MJ, Greenberg SB, Marymont JH, Shear T, Parikh KN, Patel SS, Gupta DK. Anesthesiology 2015; 122:1112-22. (Metadona intraoperatoria para la prevención del dolor postoperatorio. Un ensayo clínico aleatorizado, doble ciego en pacientes quirúrgicos cardíacos).

\section{Revisor: Evelyn Borchert. División de Anestesio- logía, Facultad de Medicina, Pontificia Universi- dad Católica de Chile.}

Introducción: La intensidad del dolor después de cirugía cardíaca a menudo es subestimado. Un control inadecuado del dolor se puede asociar con inadecuada calidad de recuperación. El objetivo de esta investigación fue examinar el efecto de la metadona intraoperatoria en los requerimientos analgésicos postoperatorios, puntajes de dolor, satisfacción del paciente y recuperación clínica.

Método: Los pacientes sometidos a cirugía cardíaca con circulación extracorpórea $(n=156)$ fueron aleatorizados para recibir metadona $(0,3 \mathrm{mg} / \mathrm{kg})$ o fentanilo $(12 \mu \mathrm{g} / \mathrm{kg})$ intraoperatoriamente. Se registraron los requerimientos analgésicos postoperatorios. Los pacientes fueron evaluados para dolor en reposo y frente a la tos a los 15 min y a las $2,4,8,12$, 24,48 y 72 h después de la extubación traqueal. Los pacientes también fueron evaluados para el nivel de sedación, náuseas, vómitos, prurito, hipoventilación e hipoxia en los momentos previamente descritos.

Resultados: El requerimiento de morfina postoperatoria durante las primeras $24 \mathrm{~h}$ se redujo de una mediana de $10 \mathrm{mg}$ en el grupo fentanilo a $6 \mathrm{mg}$ en el grupo de metadona (diferencia mediana [IC del 99\%], -4 [-8 a -2] mg; $p<0,001)$. Se observó reducción en la puntuación de dolor con tos durante las primeras 24 h después de la extubación traqueal; el nivel de dolor con tos a las $12 \mathrm{~h}$ se redujo de una mediana de 6 en el grupo fentanilo a 4 en el grupo de metadona (-2 [-3 $a-1] ; p<0,001)$. En el grupo metadona se percibió un mejor manejo del dolor. La incidencia de eventos adversos relacionados con opioides no fue mayor en el grupo metadona.

Conclusión: La administración intraoperatoria de metadona resultó en una reducción de los requerimientos de morfina postoperatoria, mejoría en los puntajes de dolor y en una mejor calidad del manejo del dolor percibida por el paciente.

Comentarios: Si bien la metadona redujo en un $40 \%$ la necesidad de morfina durante las primeras 24 $h$, la dosis efectiva no fue determinada y se compararon dos opioides que no son bioequivalentes (fentanyl versus metadona). La evaluación de dolor y las complicaciones relacionadas con los opioides solo se realizaron por $72 \mathrm{~h}$. Este estudio excluyó pacientes de cirugía cardíaca de alto riesgo, por lo que las conclusiones no pueden extrapolarse a ellos. Por último, el efecto de la metadona en el dolor crónico a largo plazo no se ha informado.

Population pharmacodynamics of propofol and sevoflurane in healthy volunteers using a clinical score and the Patient State Index. Kuizenga M, Colin P, Reyntjens K, Touw D y cols. Anesthesiology 2019: 131: 1223-38 doi: 10.1097/ ALN.0000000000002966. Farmacodinamia poblacional del propofol y sevoflurano en voluntarios sanos utilizando una puntuación clínica y el Patient State Index.

Revisor: Eduardo Vega P. División de Anestesiología, Facultad de Medicina, Pontificia Universidad Católica de Chile.

Comentario: El presente estudio describió la relación concentración-efecto de cuatro diferentes regímenes anestésicos en 36 voluntarios sanos. Investigaron cómo el remifentanil modifica las repuestas de dos hipnóticos comúnmente utilizados en una anestesia general: propofol y sevoflurano. Se evaluaron respuestas clínicas (llamar al voluntario por el nombre, la tolerancia al moverlo o gritarle y la tolerancia al estímulo tetánico (como estímulo doloroso)) y los valores de dos versiones del índice procesado del electroencefalograma (Patient State Index 1 y 2 (PSI)) que puede traducir la profundidad de hipnosis y anestesia. Lo novedoso de este estudio, con el fin de evitar las variaciones interindividuales, es que cada participante fue sometido a la administración de todas las combinaciones anestésicas de forma aleatoria, separadas por una semana. Los principales mensajes de este estudio se pueden dividir en tres preguntas y sus respuestas:

Q1: ¿Cómo afecta el remifentanil la relación entre la concentración del hipnótico y la respuesta al estímulo?

R1: El remifentanil afecta de forma similar al sevoflurano y propofol, moviendo hacia la izquierda la curva dosis/respuesta de la población (EC50). Ade- 
más, se observó que concentraciones mayores a 2 ng/ $\mathrm{ml}$ de remifentanil tendrían un beneficio decreciente en la prevención del movimiento en los pacientes que recibieron sevoflurano.

Q2: ¿El remifentanil modifica el estado de la actividad cortical a distintas concentraciones de agentes hipnóticos?

R2: Al agregar remifentanil al propofol se provocó un pequeño efecto en los valores del PSI, mientras que la adición de remifentanil al sevoflurano no causó una modificación a este. Esto podría ser explicado por las bajas concentraciones utilizadas con el propofol o bien, el escaso efecto analgésico de este.

Q3: ¿Cómo afecta el remifentanil a la relación entre el estado cortical en reposo y la probabilidad de respuesta conductual a los estímulos?

R3: La base de esta respuesta radica en el rol de los monitores EEG en la práctica clínica. La curva PSIrespuesta se desplaza a la izquierda al usar sevoflurano o adicionar remifentanil al propofol, siendo justamente lo que se ve en la práctica clínica, pudiendo dar una anestesia general fundamentalmente basada en gases, pero que en el caso del propofol este debiese ser complementado con un agente opioide cuando el paciente sea sometido a una intervención quirúrgica. Esto nos habla de que el sevoflurano es claramente mejor que el propofol para bloquear la respuesta a los estímulos externos. Sin embargo, el estado cortical, es un pobre predictor de la respuesta conductual del paciente ante un estímulo doloroso, pudiéndose provocar una respuesta motora independiente del valor que nos muestre el monitor EEG.

Queda por responder las múltiples interacciones existentes de los medicamentos que administramos de forma concomitante en pabellón como, por ejemplo, las infusiones de ketamina, dexmedetomidina o lidocaína

\section{Stochastic Pharmacokinetic-Pharmacodynamic Analysis of the Effect of Transdermal Buprenor- phine on Electroencephalogram and Analgesia. Anne Estrup Olesen, Erik Olofsen, Trine Andre- sen, Carina Graversen y cols. Anesthesia Analgesia 2015;121:1165-75). (Análisis estocástico farmacoci- netico-farmacodinámico del efecto de buprenorfina transdermica en el electroencefalograma y analgesia). \\ Revisor: Alejandro Luengo. Anestesiólogo de la División de Anestesiología. Facultad de Medici- na, Pontificia Universidad Católica de Chile.}

Introducción: El efecto de los opiodes en el tra- tamiento del dolor crónico a menudo es evaluado de forma subjetiva. En este sentido, el electroencefalograma (EEG) ofrece una posibilidad objetiva de cuantificar la actividad cerebral al administrar estas drogas. El objetivo fue investigar las propiedades farmacocinéticas y farmacodinámicas (PKPD) del parche de buprenorfina transdérmico en el EEG de reposo y la tolerancia al dolor en la piel.

Métodos: Ensayo clínico aleatorizado, doble ciego, entrecruzado, que estudió a 22 hombres sanos (edad: $23,1 \pm 3,8$ años), quienes recibieron un parche de buprenorfina transdérmico por 144 horas (20 $\mathrm{ug} / \mathrm{h}$ ) o placebo. Se midió tolerancia termalgésica en la piel del antebrazo y luego se evaluó el EEG de reposo, donde se calculó la razón de oscilaciones lentas y rápidas para un análisis posterior. A su vez se usó un modelo poblacional PKPD con una ecuación diferencial estocástica para la absorción de la droga desde el parche y analizar simultáneamente los datos PK y PD utilizando el paquete estadístico NONMEM.

Resultados: Buprenorfina aumenta la razón del EEG $(P=0,0006)$ y la tolerancia al dolor de la piel ( $P$ $=0,0008)$ comparado con placebo. El modelo estocástico caracteriza adecuadamente la concentración y el efecto en el tiempo para ambos desenlaces con variaciones en la tasa de absorción de la droga durante las 144 horas de tratamiento. Como parámetro de potencia, el efecto en el EEG fue 10 veces más sensible al efecto de buprenorfina que el test de dolor cutáneo.

Comentarios: Usando un análisis estocástico PKPD, se cuantificó exitosamente el efecto del parche de buprenorfina sobre el EEG de reposo y la tolerancia al dolor cutáneo. Ambos desenlaces fueron afectados por esta droga, aunque el EEG de reposo fue más sensible. El análisis estocástico PKPD permite calcular la variabilidad en el tiempo de la absorción de la droga desde el parche a la sangre. La información sugiere que el EEG de reposo es una atractiva y objetiva alternativa para evaluar el efecto opioide.

En este sentido se puede establecer que el EEG es una herramienta útil para evaluar la acción de una droga cuyo efecto hasta este momento era determinado por métodos subjetivos y en los que la dosificación es siempre desafiante. A su vez, el hecho de modelar mediante una ecuación diferencial el cambio de la absorción de la droga en el tiempo, explicado por las diferencias en el flujo sanguíneo cutáneo diurno, permiten dar una mejor aproximación a las concentraciones reales de la droga en la sangre. De esta forma se puede tomar precauciones acerca de los determinantes que podrían aumentar su absorción.

Sin duda que el uso de un modelo de absorción y 
distribución en conjunto con el EEG surge como una nueva herramienta que permite comprender de mejor forma tanto la dosificación (PK) como la evaluación del efecto (PD) del parche de buprenorfina. De esta forma, se pueden incorporar y explicar variables que afectan al modelo y la concentración sanguínea de la droga como son las diferencias de perfusión cutánea dadas, por ejemplo, por la temperatura de la piel. Pese a este importante hallazgo, aún queda por determinar si la evaluación del EEG se ve afectada en presencia de otras drogas que actúen a nivel del SNC y establecer una escala dosis-respuesta para acercarse con mayor precisión a los requerimientos de analgesia de cada paciente.

Damian MA, Hammer GB, Elkomy MH, Frymoyer A, Drover DR, Su F. Pharmacokinetics of Dexmedetomidine in Infants and Children After Orthotopic Liver Transplantation. Anesth Analg. 2020 Jan;130(1):209-16. (Farmacocinética de la dexmedetomidina en lactantes y niños después de trasplante hepático ortotópico).

Revisor: Héctor J. Lacassie. División de Anestesiología, Facultad de Medicina, Pontificia Universidad Católica de Chile.

Introducción: La dexmedetomidina (DEX) es un medicamento sedante y analgésico que se usa frecuentemente en el postoperatorio en niños después de trasplante hepático. La disfunción hepática, incluidas las alteraciones en la eliminación de fármacos, es común inmediatamente postrasplante. Se desconoce la farmacocinética (PK) de DEX en esta población. El objetivo fue determinar el perfil PK de DEX en niños después del trasplante hepático.

Métodos: Estudio PK poblacional unicéntrico de DEX administrada como una dosis de carga intravenosa de $0,5 \mu \mathrm{g} / \mathrm{kg}$ seguido de una infusión continua de $0,5 \mu \mathrm{g} / \mathrm{kg} / \mathrm{h}$. Se estudiaron veinte niños de 1 mes a 18 años de edad, que ingresaron en la Unidad de Cuidados Intensivos Pediátricos postrasplante. Se analizó la concentración de DEX usando un método de gota de sangre seca en papel secante. Se realizó un modelamiento de efectos mixtos no lineales para caracterizar la PK poblacional de DEX.

Resultados: La PK de DEX fue mejor descrita por un modelo de 2 compartimentos con eliminación de primer orden. Un típico niño postrasplante hepático con una razón internacional normalizada (INR) de 1,8 tiene: clearance total: $52 \mathrm{~L} / \mathrm{h}$ (IC 95\%, 31-73 L/h); clearance intercompartimental: 246 L/h (IC 95\%,
139-391 L/h), volumen de distribución 1 (central): 186 L/70 kg (IC 95\%, 140-301 L/70 kg) y volumen de distribución 2 (periférico): 203 L (IC 95\%, 123-338 L). La variabilidad interindividual varió de $11 \%$ a $111 \%$ para todos los parámetros. El clearance total fue inversamente proporcional al INR, que fue usado como parámetro subrogante de función hepática. El peso se correlacionó linealmente (no alométricamente) con el volumen central de distribución.

Conclusiones: Niños que recibieron Dex post trasplante hepático tienen alta variabilidad en el clearance, que no se asocia al peso, sino que a la función hepática representada por el INR. La titulación de Dex a efecto clínico es importante en esta población.

Comentarios: Se trata de un estudio más de PK poblacional, donde la validación del método de medición utilizado (gota de sangre) abre una nueva veta en la investigación. Si bien la variabilidad encontrada es muy alta, los datos siguen siendo valiosos. Algunos inconvenientes del estudio son que se hace difícil comparar los resultados con otros estudios en que se utilizan niveles plasmáticos de Dex; y que no se exploraron otros factores que podrían alterar la PK de la Dex (como la masa hepática trasplantada o la etnia), principalmente por un número reducido de pacientes reclutados.

Joosten A, Rinehart J, Bardaji A, y cols. Anesthetic Management Using Multiple Closed-Loop Systems and Delayed Neurocognitive Recovery. Anesthesiology 2020;132(2):253-266. (Manejo anestésico usando sistemas múltiples de circuito cerrado y recuperación neurocognitiva tardía).

Revisor: Daniela Muñoz Biggs. División de Anestesiología, Facultad de Medicina, Pontificia Universidad Católica de Chile.

Introducción: Los sistemas de circuito en asa cerrada han mostrado consistentemente mejorar la calidad en la administración de drogas y fluidos en comparación con la administración manual. Se postula que el manejo de 1) profundidad anestésica; 2) administración de fluidos y 3) ventilación pulmonar bajo 3 sistemas independientes de circuito cerrado superaría el control manual de estas variables y como resultado, los pacientes presentarían menor deterioro neurocognitivo postoperatorio que pacientes sometidos a anestesia bajo control manual.

Materiales y Métodos: Estudio clínico unicéntri$\mathrm{co}$, aleatorizado, doble ciego, que incluyó 90 pacientes sobre 60 años ingresados para cirugía electiva no 
cardíaca, de riesgo intermedio y alto, bajo anestesia total intravenosa por TCl. Hubo criterios explícitos de inclusión y exclusión. Grupo control: titulación manual de la anestesia (propofol y remifentanil para un BIS 40-60), fluidos (a criterio del tratante) y ventilación (a criterio del tratante). Grupo asa cerrada: la titulación de la anestesia (guiada por BIS), fluidos (protocolo por objetivos y guiado por volumen sistólico) y ventilación (estrategia protectora, guiada por objetivo de $\mathrm{EtCO}_{2}$ ) se hizo con 3 sistemas independientes de circuito en asa cerrada. Se realizó evaluación neurocognitiva (test de MoCA y otros) por un equipo especializado y ciego el día anterior a la cirugía (día 1), durante la primera semana del postoperatorio y a los 3 meses. El desenlace primario fue: cambios neurocognitivos el día 1 y la semana posterior a la cirugía. Los desenlaces secundarios fueron: cambio neurocognitivos el día 1 y 3 meses, calidad de vida y de recuperación, dosis total de drogas y de fluidos, parámetros de monitorización en rango normal, complicaciones durante hospitalización, tiempo de estadía en unidad de recuperación o UPC y mortalidad a 1 y 3 meses.

Resultados: El grupo control disminuyó en 1 su puntaje neurocognitivo en la primera semana, sin cambios en el grupo intervencional (-1 [-2 a 0] vs 0 [-1 a 1]; diferencia de 1 [IC 95\%, 0 a 3], $P=0,033$ ) lo que se mantuvo a los 3 meses.

Pacientes en el grupo intervencional estuvieron menor tiempo con índice BIS bajo 40 y un mayor tiempo con índice BIS en rango (40-60). Además, presentaron menor balance hídrico menor y tiempo de $\mathrm{EtCO}_{2}$ bajo 32 y mayor tiempo de mantención con $\mathrm{EtCO}_{2}$ en objetivo (32-38). Asimismo, requirieron menos dosis totales de propofol y remifentanil y menores ajustes de dosis. Por último, no hubo diferencias significativas en tiempos de estadía, complicaciones y mortalidad en ambos grupos.

Conclusiones: En pacientes mayores, no frágiles, sometidos a cirugía no cardíaca de riesgo intermedio y alto, un tratamiento anestésico automatizado, que utiliza la combinación de tres sistemas independientes de circuito en asa cerrada superó el control manual y podría tener un impacto en la recuperación neurocognitiva tardía.

Discusión: Estudios previos en sistemas de circuito en asa cerrada muestran una mejora en la calidad de administración de drogas y fluidos, algo que hasta el momento no se había podido demostrar en los desenlaces postoperatorios. Este es el primer estudio que muestra un cambio significativo en el postoperatorio, además, de lograr utilizar tres sistemas independientes de circuito en asa cerrada, lo que es novedoso. Sin embargo, es discutible si el cambio en 1 de punto en el puntaje neurocognitivo es clínicamente relevante. Ahora bien, este cambio fue sostenido por 3 meses y podría ser muy invalidante para los pacientes. El diseño del estudio no permite sacar conclusiones respecto al impacto individual de cada sistema en forma independiente. Por último, se necesitan futuros estudios que incluyan pacientes frágiles y con peor estado neurocognitivo previo con el objetivo de evidenciar un tamaño de efecto potencialmente mayor.

The Human Burst Suppression Electroencephalogram of Deep Hypotermia. Westover MB, Ching S, Kumaraswamy VM, Akeju SO, Pierce E, Cash SS, Kilbride R, Brown EN, Patrick LP. Clin Neurophysiol. 2015 October; 126(10): 1901-1914. doi:10.1016/j.clinph.2014.12.022 (El electroencefalograma humano de brotes y supresiones en la hipotermia profunda).

Revisor: Dra. Evelyn Borchert. División de Anestesiología, Facultad de Medicina, Pontificia Universidad Católica de Chile.

Introducción: La hipotermia profunda induce "brotes y supresiones" (burst supressions (BS)), un patrón electroencefalográfico con "supresiones" de bajo voltaje que se alternan con "brotes" de alto voltaje. La comprensión actual de BS proviene principalmente de estudios realizados en anestesia, mientras que la BS inducida por hipotermia no ha sido muy estudiada. El objetivo fue investigar los cambios en el electroencefalograma inducidos por el enfriamiento del cerebro humano a través de profundidades crecientes de BS hasta la isoelectricidad (inactividad electroencefalográfica).

Método: Se registraron electroencefalogramas (EEG) del cuero cabelludo de once pacientes sometidos a hipotermia profunda durante una cirugía cardíaca con paro circulatorio y se analizaron utilizando métodos de análisis espectral.

Resultados: La profundidad de BS depende sistemáticamente de la profundidad de la hipotermia, aunque las respuestas varían entre los pacientes, excepto a temperaturas extremas. Con la disminución de la temperatura, las longitudes de las supresiones aumentan y las amplitudes y longitudes de los brotes disminuyen, mientras que el contenido espectral (morfología) de los estallidos permanece constante.

Conclusión: Estos hallazgos respaldan un modelo teórico existente en el que el mecanismo común de BS en diversas etiologías es el agotamiento difuso cíclico de los recursos metabólicos y sugieren la nue- 
va hipótesis de "deserción de la microred local" para explicar la disminución de las amplitudes de brotes a temperaturas más bajas.

Comentarios: Estos resultados allanan el camino para un seguimiento preciso y no invasivo del estado metabólico cerebral durante los procedimientos quirúrgicos bajo hipotermia profunda y sugieren nuevas predicciones comprobables sobre los mecanismos de red subyacentes de BS. El seguimiento de la morfología espectral de los brotes durante la hipotermia pro- funda permitiría controlar si la dinámica del circuito neural subyacente permanece intacta. La pérdida o las alteraciones del contenido espectral normal dentro de los brotes pueden servir como un indicador sensible de lesión cerebral isquémica-anóxica. El monitoreo continuo de EEG para detectar la evolución a lo largo del tiempo de la morfología de los brotes hacia un patrón normal podría ser útil como un medio sensible para detectar la recuperación de una lesión o la resolución de la patología. 